\title{
Genetically-Tunable Mechanical Properties of Bacterial Functional Amyloid Nanofibers
}

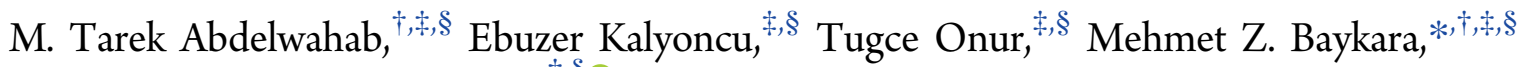 \\ and Urartu Ozgur Safak Seker*,+, ${ }^{*}$ \\ ${ }^{\dagger}$ Department of Mechanical Engineering, Bilkent University, Ankara 06800, Turkey \\ ${ }^{\ddagger}$ Institute of Materials Science and Nanotechnology, Bilkent University, Ankara 06800, Turkey \\ ${ }^{\S}$ National Nanotechnology Research Center (UNAM), Bilkent University, Ankara 06800, Turkey
}

\section{Supporting Information}

\begin{abstract}
Bacterial biofilms are highly ordered, complex, dynamic material systems including cells, carbohydrates, and proteins. They are known to be resistant against chemical, physical, and biological disturbances. These superior properties make them promising candidates for next generation biomaterials. Here we investigated the morphological and mechanical properties (in terms of Young's modulus) of genetically-engineered bacterial amyloid nanofibers of Escherichia coli (E. coli) by imaging and force spectroscopy conducted via atomic force microscopy (AFM). In particular, we tuned the expression and biochemical properties of the major and minor biofilm proteins of E. coli (CsgA and CsgB, respectively). Using appropriate mutants, amyloid nanofibers constituting biofilm backbones are formed with different combinations of CsgA and CsgB, as well as the optional addition of tagging sequences. AFM imaging and force spectroscopy are used to probe the morphology and measure the Young's moduli of biofilm protein nanofibers as a function of protein composition. The obtained results reveal that genetically-controlled secretion of biofilm protein components may lead to the rational tuning of Young's moduli of biofilms as promising candidates at the bionano interface.
\end{abstract}

\section{INTRODUCTION}

Viral capsid proteins, ${ }^{1,2}$ cage proteins, ${ }^{3,4}$ bacterial microcompartments, ${ }^{5}$ filamentous bacteriophage coats, ${ }^{6}$ bacterial flagella, ${ }^{7}$ bacterial pili, ${ }^{8}$ and amyloids ${ }^{9}$ are the key self-assembled protein nanostructures. Among these, amyloid proteins are characterized as durable protein structures under harsh physical and chemical conditions such as low $\mathrm{pH}$, extreme temperatures, or proteolysis. ${ }^{10}$ Amyloids can be classified into two groups, namely, pathological amyloids ${ }^{11}$ and functional amyloids. ${ }^{12}$ Functional amyloids are those synthesized, mostly, by bacteria during the formation of the biofilm structures, ${ }^{13}$ whereas pathological amyloids are formed as result of protein aggregation observed during neurodegenerative disease conditions. ${ }^{14}$ Functional amyloids are a crucial part of biofilm structures as they form its backbone. Within the bacterial biofilm structure, functional amyloids are capable of forming long-range wire-like assemblies resulting in fibril networks. ${ }^{15}$ Although biofilms are mostly associated with infectious disease conditions, functional amyloids forming these complex structures offer new capabilities and functionalities as a new generation of biomaterials with high durability and ease of functionalization through genetic engineering and protein engineering approaches. ${ }^{16,17}$ Deliberate utilization of biofilm proteins as functional materials is in its early stages. As such, there is a strong need to understand the mechanical, chemical, and biological properties of these systems for further utilization. ${ }^{18}$ One of the unique opportunities presented by protein-based materials is the fact that their biochemical properties can be tuned through common protein engineering and genetic engineering practices. These practices can lead to the formation of well-controlled, tunable, regenerative material systems. $^{19-22}$

Biofilm formation is a common phenomenon for many bacteria including Escherichia coli (E. coli). E. coli biofilm forming proteins, namely, CsgA and CsgB, are controlled by an operon called the curli operon. Curli operon controls the biofilm formation under stress conditions and switches from planktonic phase into biofilm phase. During this phase switch, two major curli operon proteins forming the biofilm backbone and curli fibers, namely, CsgA and CsgB, are secreted and transported through the cell membrane with the help of accessory proteins CsgE, CsgF, and CsgG. ${ }^{15}$ CsgB serves as the nucleator protein for CsgA to grow the fiber network. ${ }^{23}$ Studies were carried out on the practical utilization of curli fibers. In a recent work,

Received: January 12, 2017

Revised: March 23, 2017

Published: April 7, 2017 
utilization of the curli fiber for programmed patterning of the nanomaterials was investigated, where the curli fiber forming CsgA protein was expressed from the cells in a programmed manner. ${ }^{16}$ Fusion of curli fiber with well-known mussel adhesive proteins was demonstrated as an underwater adhesive application in a subsequent study. ${ }^{21}$ These studies were promising toward understanding of the potential of the curli fibers and biofilm matrix as new generation biomaterials. Despite the few efforts discussed above, there is a need for a thorough characterization of biofilm protein backbones in order to realize their utilization as biomaterial elements for functional uses. Among the potential functional studies of biofilms, probing their mechanical properties is of particular importance. Atomic force microscopy (AFM), since its invention in 1986, ${ }^{24}$ has been rapidly adopted by the biology community for the high-resolution imaging of biological materials as well as the measurement of associated mechanical properties via force spectroscopy. ${ }^{25-29}$ The main advantage of AFM with respect to electron microscopy in biology research is the fact that it does not require vacuum to operate ${ }^{30,31}$ and thus may be used to study biological materials under ambient and liquid environments that correspond to near-native conditions, which in turn allows the samples to preserve their structure and functionality. For instance, AFM has been successfully used to study in highresolution individual cells, ${ }^{32,33}$ single proteins, $^{34}$ genetic material such as chromosomes, chromatinm and DNA, ${ }^{35}$ and cell membranes, ${ }^{36}$ among other biological materials. Moreover, via force-distance (FD) spectroscopy, AFM has been employed to differentiate between healthy and cancerous cells. ${ }^{37}$ AFM-based FD spectroscopy can also be used to measure interactions between antibiotics and bacterial cell walls $^{38}$ as well as the elastic properties of individual cells. ${ }^{39}$ Moreover, the recent development of high-speed $\mathrm{AFM}^{40}$ now allows researchers to track biological processes ${ }^{41}$ and measure mechanical properties of biological materials, ${ }^{42}$ not only with outstanding resolution in space but also in time. On the other hand, AFM-based studies of mechanical properties associated with amyloid nanofibers are quite limited. ${ }^{43}$ In particular, the strongly anisotropic mechanical properties of individual insulin amyloid nanofibers have been previously probed via FD experiments. ${ }^{44,45}$ FD experiments have also been employed to study the mechanical properties of biofilm-producing bacteria, but not the amyloid fibers forming the backbone of the biofilm itself. $^{46,47}$

In the case of curli-based materials (such as bacterial functional amyloid nanofibers), mechanical properties of the final material assembly will be controlled by the CsgA and CsgB proteins forming the curli fibers. Based on this fact, upon adjusting the secretion of these proteins in a controlled manner, the associated Young's moduli of the amyloid nanofibers have been compared and contrasted via AFM in this study. Additionally, experiments have been conducted to understand the effect of fusing soft Linkers involving aromatic rings containing histidine tag with CsgA and CsgB proteins on Young's modulus. AFM imaging experiments also allowed a comparative and qualitative study of the morphology associated with the structures formed by specific amyloid nanofibers. Overall, the results reported here lead to the conclusion that curli fibers can be genetically tuned to provide stiffer or softer amyloid nanofiber structures. These findings support the idea of the utilization of curli fibers as protein polymers in functional applications aimed at forming composite materials with well- defined mechanical properties, which can be tuned easily via genetic engineering and protein engineering approaches.

\section{EXPERIMENTAL DETAILS}

2.1. Preparation of Genetic Constructs and Cloning of $\operatorname{csg} A$ and $\operatorname{cs} g B$. Gene fragments were amplified from genomic DNA of Escherichia coli DH5a. csgA gene fragments were amplified by using the primers listed in Table $S 1$ in the Supporting Information. F1-R1 primers were used to amplify CsgA and F2-R2 primers were used to amplify CsgB; their sequences are listed in Table S2 in the Supporting Information. Recombinant gene fragments were cloned into $\mathrm{pZa}$ $\mathrm{CmR}-\mathrm{TTc}$-ribo vector and $\mathrm{pZa}-\mathrm{AmR}$-aTc-ribo vector with cut ligate method by using kpnI and mluI endonucleases (NEB). Recombinant plasmids were isolated with QIAprep Spin Miniprep Kit. Plasmid constructs were sequence-verified by Genescript. In addition, $\operatorname{csg} A$ fragments amplified from Escherichia coli DHSa genome and soft Linker sequence (3xGGGS), TEV protease restriction site, and soft Linker sequence (GGGS) were added to $3^{\prime}$ prime of the wild type $\operatorname{csg} A$ gene with two PCR reactions. Then, PCR products were cloned into $\mathrm{pET} 22 \mathrm{~b}(+)$ expression vector. NcoI and XhoI (NEB) endonucleases were used in digestion reactions, and T4 ligase (NEB) enzyme was used in the ligation reaction. QIAprep Spin Miniprep Kit was used to isolate plasmids, and they were verified by Genewiz. In order to clone $\operatorname{csg} B$ gene, $\operatorname{csg} A$ gene fragments were extracted from the new recombinant vector by using $\mathrm{NcoI}$ and $K p n \mathrm{I}$ (NEB) endonucleases. F3-R3 and F4-R4 primers were used to construct the modified CsgAM protein coding gene fragments, and cloning steps were achieved as described above. Genes coding for CsgBM protein was prepared using F5-R5 and F6-R6 primers. The sequence information on the primers is given in Table S1.

2.2. Biofilm Formation Using Engineered Constructs. Our inducible synthetic circuits were transformed to E. coli MG1655 PRO $\triangle \operatorname{csgBA}$ ompR234 host strain. $\operatorname{csg} A$ and $\operatorname{csgB}$ genes were knocked out in this strain. For the starting culture, a single colony was inoculated to $\mathrm{LB}$ medium at $37^{\circ} \mathrm{C}$ for $16 \mathrm{~h}$. For the induction of biofilm formation, anhydrotetracycline (aTc) or IPTG were added into M63 minimal medium supplemented with $1 \mathrm{mM} \mathrm{MgSO}_{4}$ and $0.2 \%$ glucose, and cultures were grown at $30{ }^{\circ} \mathrm{C}$ in 24 -well plates without shaking for 3 days.

2.3. Congo Red Staining of Curli Structures. In order to assay the formation of the functional amyloids in situ, a single colony was inoculated into $3 \mathrm{~mL}$ of LB including relevant antibiotic and grown at $37^{\circ} \mathrm{C}$ for $16 \mathrm{~h}$. Then, $0.34 \mathrm{~mL}$ of overnight culture was transferred into $10 \mathrm{~mL}$ of LB. Induction of curli fibers was performed with aTc (final concentration of $200 \mathrm{ng} / \mu \mathrm{L}$ ) or IPTG (final concentration of 1 $\mathrm{mM}$ ) for $24 \mathrm{~h}$. A $900 \mu \mathrm{L}$ aliquot of cell culture was mixed with $5 \mathrm{X}$ Congo red solution (final concentration of $25 \mu \mathrm{g} / \mathrm{mL}$ ) and incubated for $30 \mathrm{~min}$ at room temperature. For the quantification of curli-bound Congo red (CR), cells were centrifuged at $14000 \mathrm{rpm}$ for $5 \mathrm{~min}$. The absorbance of $25 \mu \mathrm{g} / \mathrm{mL} \mathrm{CR}$ was subtracted from the absorbance of supernatant at $480 \mathrm{~nm}\left(\mathrm{AbsCR}_{\mathrm{OD} 480}\right)$ and normalized for total cell concentration $\left(\mathrm{AbsCR}_{\mathrm{OD} 600}\right)$. The calculation of the amount of $\mathrm{CR}$ bound cells and curli fibers was performed via the following expression: $-\mathrm{AbsCR}_{\mathrm{OD} 480} / \mathrm{AbsCR}_{\mathrm{OD} 600}$.

2.4. SEM Imaging of Curli Structures. After the formation of biofilms on 24-well plates, M63 minimal medium of the wells were removed. Cells were washed two times with phospahate-buffered saline (PBS). Then, cells were fixed on silicon wafer with $2.5 \%$ gluteraldehyde solution in PBS overnight at $+4{ }^{\circ} \mathrm{C}$. After fixing cells, dehydration was performed via serial ethanol washing steps with increasing concentration. Samples were dried at room temperature and were sputtered with $5 \mathrm{~nm} \mathrm{Au} / \mathrm{Pd}$ alloy. FEI Quanta 200 FEG scanning electron microscopy was used for imaging.

2.5. AFM Imaging and Characterization of Young's Moduli of the Amyloid Nanofiber Structures. High-resolution imaging (in contact mode) and FD experiments were conducted under ambient conditions (temperature, $21 \pm 1{ }^{\circ} \mathrm{C}$; relative humidity, $25 \pm 5 \%$ ) using a commercial AFM instrument (Nanomagnetics Inc.). A single AFM probe (Nanosensors PPP-CONTR) with a spring constant of $0.20 \mathrm{~N} /$ 


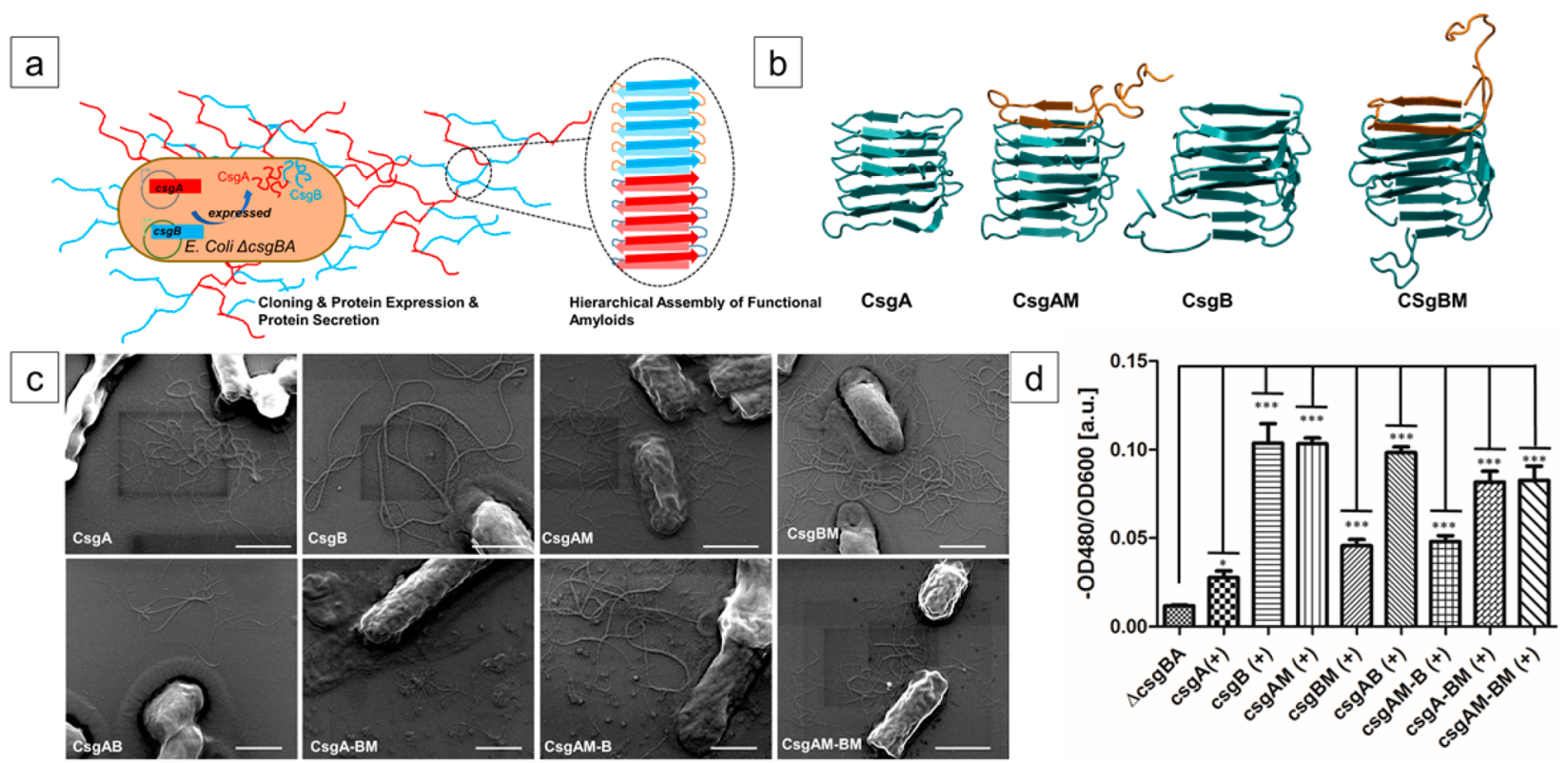

Figure 1. Secretion of the functional amyloid nanofibers through the cellular machine and the qualitative as well as quantitative validation of the secretion of the amyloid nanofibers. (a) Graphical representation of the cloning of $\operatorname{Csg} A$ and $C s g B$ genes into the expression vectors, the transformation of $\Delta \operatorname{csg} B A$ cells with cloned vectors, followed by the secretion of soluble CsgA and CsgB proteins (and their modified versions) into the extracellular environment and the formation of the functional amyloid backbone of the biofilm. (b) Molecular models of CsgA, CsgAM, and $\mathrm{CsgB}$, CsgBM predicted using the Raptor X program. Orange parts represent Linker amino acid sequences. (c) SEM images of the biofilm protein backbones formed using the genetic constructs. These constructs are used for AFM characterization. The scale bar is $1 \mu \mathrm{m}$. (d) Congo red assay results representing amyloid formation $(p<0.05)$. One-way analysis of variance test was used to compare all samples with the control sample $(*$, significant; $* * *$, extremely significant).

$\mathrm{m}$ (determined via the Sader method $^{48}$ ) has been utilized for all experiments. The geometry of the AFM probe apex has been determined via scanning electron microscopy (SEM; see the Supporting Information). All samples have been prepared by dropcasting on freshly cleaved mica substrates and subsequent drying under ambient conditions. The experimental procedure associated with the use of FD curves toward the determination of Young's modulus values is explained in detail in the Supporting Information. In summary, a series of FD curves are acquired on each sample to be investigated, where curves acquired on the cells themselves and the mica substrate are used as control experiments. Each curve is linearly fitted, and the corresponding equivalent stiffness (with contributions from both the sample and the cantilever) was calculated. Indentation into the sample was determined by comparison with FD curves acquired on the hard mica substrate, and subsequently, force-indentation curves were extracted. To determine the Young's moduli from these data, the Hertzian contact model was used, taking into account the flat-ended geometry of the specific tip apex employed in the experiments.

2.6. Molecular Modeling of the Proteins. The CsgA and CsgB proteins and their derivatives with linker sequences are modeled via RaptorX web-based 3D protein structure prediction tool, which generates multiple structures. ${ }^{49}$ Among the multiple structures generated by the Raptor X algorithm, the model that has the highest score, which also coincides with the previously generated model in the literature, was selected. ${ }^{50}$

\section{RESULTS AND DISCUSSION}

3.1. Morphology of Amyloid Nanofiber Structures. The route of secretion of the biofilm proteins and formation of the functional amyloid backbone is presented in Figure 1a. CsgA and CsgB proteins have different polymerization routes and different morphologies. We expect that these morphological and biochemical differences may lead to a change in overall mechanical properties of the fibers. In order to control the mechanical properties of biofilm protein fibers, one needs to understand the mechanical properties of individual fibers formed by CsgA and CsgB proteins and their "modified" versions. CsgA and $\mathrm{CsgB}$ proteins were modified by adding Linker molecules as described in the Supporting Information. The predicted molecular structures of CsgA and CsgB and their modified versions showed different structural features as presented in Figure 1b. It is also obvious that there is more structural disturbance in CsgB compared to CsgA upon the addition of the Linker molecule. This rough observation also gives a clue about variations in the Young's moduli of the protein fibers reported in this work.

In order to study the potential tunability of the Young's moduli of amyloid nanofibers associated with bacterial biofilms, a comparative study has been designed that involves three different groups of samples. We probe the change in the Young's modulus of the CsgA and CsgB proteins and their mixture $(\mathrm{Csg} A B)$ upon addition of soft Linker molecules as a tuning element to the $E$. coli (already knocked out of its native CsgA and CsgB proteins to constitute a control sample). In the native biofilm structure, CsgA and CsgB proteins are both found; however, in our case, to be able to tune the overall fiber properties in terms of Young's modulus, we need to understand the individual properties of the cell-secreted proteins. To do so, eight different samples have been generated, which are categorized into three different groups: Normal (CsgA, CsgB, and CsgAB), Linker-modified (CsgAM, CsgBM, and CsgAM$\mathrm{BM}$ ), and Complementary (CsgAM-B and CsgA-BM). All of these proteins were secreted into the extracellular space, and samples were investigated with SEM to validate the secretion of the proteins; related images are shown in Figure 1c. Functional amyloid formation is validated with a $\beta$-sheet specific dye molecule called Congo red (CR). ${ }^{51}$ Quantitative Congo red assay experiments were performed to confirm the formation of 

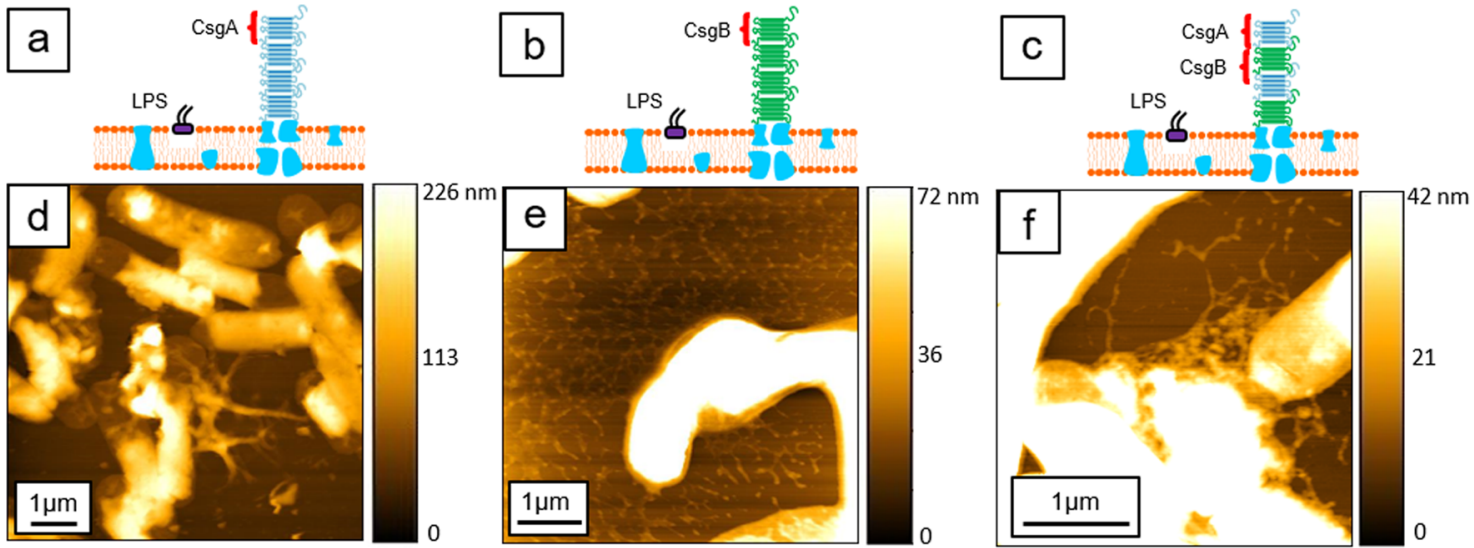

Figure 2. Morphology of samples in the Normal group as imaged by AFM. Structural schematics corresponding to each sample (CsgA, CsgB, and $\mathrm{Csg} A B)$ are depicted in panels a-c, respectively. Topographical AFM images for each sample (CsgA, CsgB, and CsgAB) as well as scale bars are shown in panels $\mathrm{d}-\mathrm{f}$, respectively.

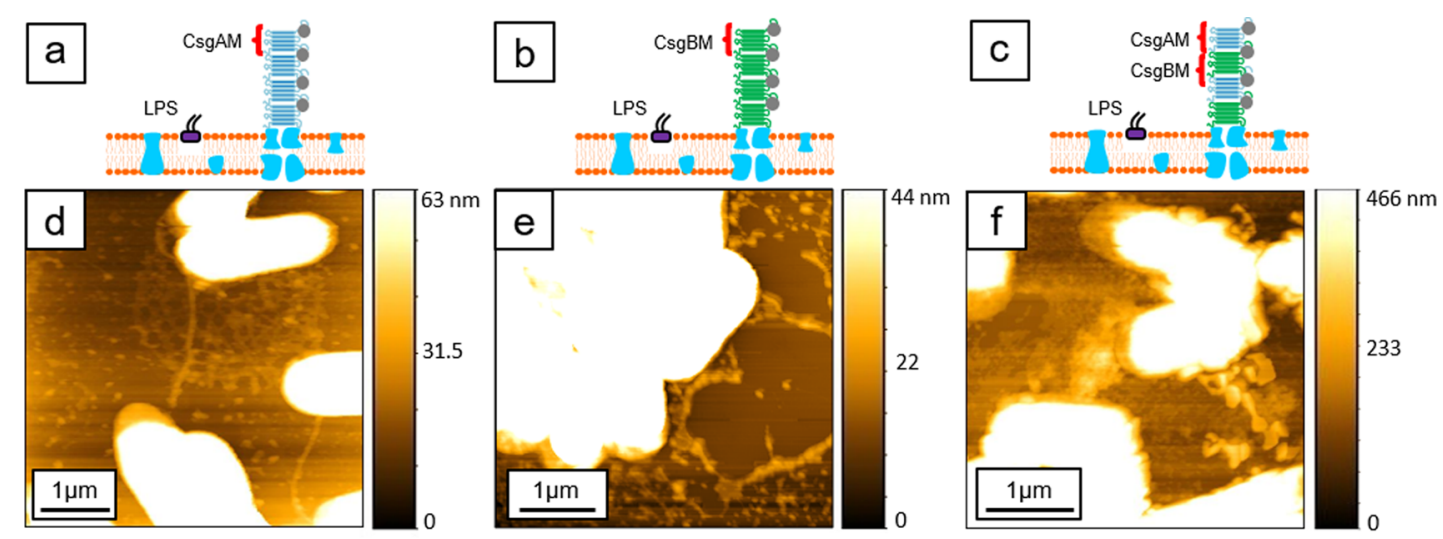

Figure 3. Morphology of samples in the Linker-modified group as imaged by AFM. Structural schematics corresponding to each sample (CsgAM, CsgBM, and CsgAM-BM) are depicted in panels a-c, respectively. Topographical AFM images for each sample (CsgAM, CsgBM, and CsgAM-BM) as well as scale bars are shown in panels $\mathrm{d}-\mathrm{f}$, respectively.

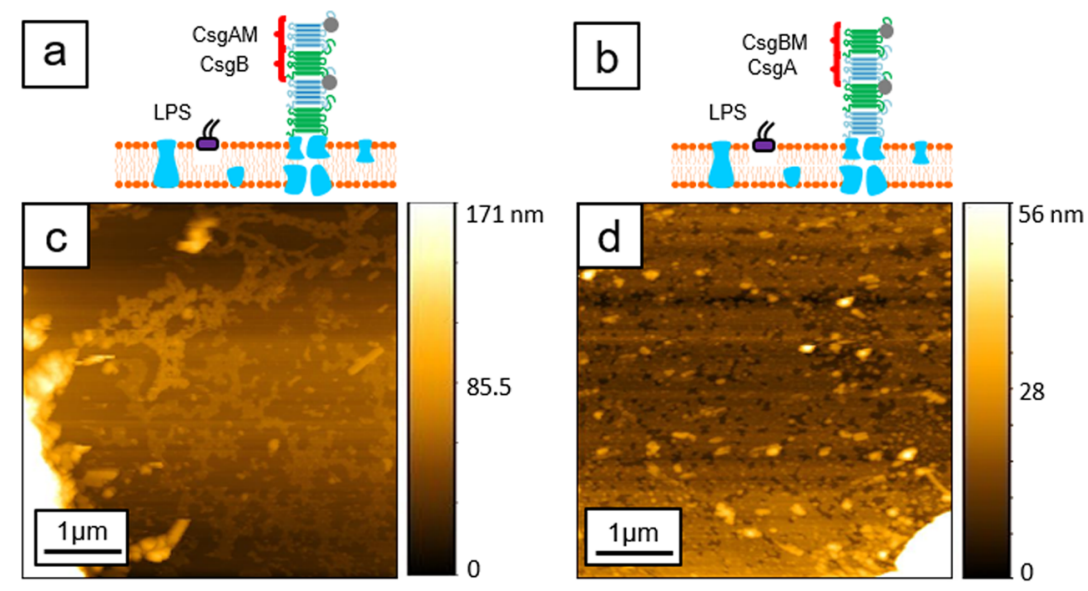

Figure 4. Morphology of samples in the Complementary group as imaged by AFM. Structural schematics corresponding to each sample (CsgAM-B and CsgA-BM) are depicted in panels a and b, respectively. Topographical AFM images for each sample (CsgAM-B and CsgA-BM) as well as scale bars are shown in panels $\mathrm{c}$ and $\mathrm{d}$, respectively.

curli fibrils produced by recombinant bacteria; the related results are shown in Figure 1d. All of these recombinant bacteria produce curli fibers to a greater extent than $E$. coli $\Delta c s g B A$ cells. CsgA, CsgBM, and CsgAM-CsgB samples produced a lower amount of curli fibers than the rest of the samples.
The schematics in Figures 2, 3, and 4 depict the basic structure of the amyloid fibers which are composed of CsgA and $\mathrm{Csg} B$ proteins, shown as blue and green sheets, respectively, as well as silver dots linked to the protein sheet (where applicable), denoting the Linker sequences. The morphological characterization of the different samples has 
been performed by AFM imaging experiments, as shown by the respective images in Figures 2-4.

Panels $\mathrm{d}-\mathrm{f}$ of Figure 2 depict the morphology of the samples associated with the Normal group: The CsgA sample (Figure 2d) features isolated nanofiber structures with limited lateral extent around the bacteria. On the other hand, the nanofibers of the CsgB sample (Figure 2e) form porous networks. Finally, the last sample in this group (CsgAB) roughly mimics the morphology of the CsgA sample (Figure 2f).

Panels $\mathrm{d}-\mathrm{f}$ of Figure 3 depict the morphology of the samples associated with the Linker-modified group While the nanofiber structures associated with the CsgAM (Figure 3d) and CsgBM (Figure 3e) samples resemble each other, with porous networks forming around the bacteria, the CsgAM-BM sample results in a much more continuous distribution of nanofibers on the substrate surface (Figure 3f).

Lastly, panels c and d of Figure 4present the morphology of the samples in the Complementary group, where only one of the two proteins (CsgA or CsgB) is Linker-modified. As one can see in Figure 4c, the CsgAM-B sample features amyloid nanofibers bursting out of bacteria that form large-extent, porous networks. On the other hand, for the CsgA-BM sample shown in Figure 4d, the fibers have merged to form a continuous. long-range, film-like structure around the bacteria with very low porosity. It is to be noted that the mean values of the nanofiber height for each sample were determined as follows: CsgA $(60 \mathrm{~nm}), \operatorname{CsgB}(13.5 \mathrm{~nm}), \operatorname{CsgAB}(9 \mathrm{~nm})$, CsgAM (5 nm), CsgBM (12.5 nm), CsgAM-BM (17.5 nm), CsgAM-B $(12.5 \mathrm{~nm})$, and CsgA-BM $(7.5 \mathrm{~nm})$. On the other hand, no significant correlation between different types of samples and associated heights has been found in the context of expected fiber structure.

3.2. Tunable Young's Modulus of Amyloid Nanofiber Structures. Following the investigation of the morphology associated with amyloid nanofiber structures via AFM imaging as described in the previous section, multiple FD spectroscopy experiments have been performed on each of the investigated samples to extract mean Young's modulus $\left(E_{\text {mean }}\right)$ and standard error of the mean (SEM) values (for details of the measurement procedure, please see the Supporting Information; indentation points on amyloid nanofibers are demonstrated on a sample of AFM images in Figure S4). The results of these experiments are summarized in Table 1 and Figure 5, which shows histograms of Young's moduli for each sample group (Normal, Linker-modified, and Complementary). Figure 5a was prepared using two-way ANOVA's comparisons of CsgA, CsgAM, CsgB, CsgBM, and CsgAB samples. Figure 5b was

Table 1. Young's Modulus (Mean and Standard Error of Mean) Values for All Samples Classified in the Normal, Linker-Modified, and Complementary Groups

$\begin{array}{ccc}\text { amyloid nanofiber samples } & E_{\text {mean }}(\mathrm{MPa}) & \text { SEM }(\mathrm{MPa}) \\ \text { CsgA } & 9.5 & 1.4 \\ \text { CsgB } & 10.0 & 0.7 \\ \text { CsgAM } & 8.4 & 0.8 \\ \text { CsgBM } & 13.9 & 0.8 \\ \text { CsgAB } & 11.6 & 0.8 \\ \text { CsgAM-BM } & 14.8 & 0.8 \\ \text { CsgAM-B } & 10.4 & 0.6 \\ \text { CsgA-BM } & 18.9 & 1.0\end{array}$

prepared using one-way ANOVA's comparisons of CsgAB, CsgAM-B, CsgA-BM, and CsgAM-BM samples.

The values reported in Table 1 and Figure 5 reveal that the rational tuning of the mechanical properties of the amyloid nanofibers in terms of stiffness (as assessed by $E_{\text {mean }}$ ) can be achieved via genetic engineering. The first four samples in Table 1 are the main constituents from which the rest of the samples are constructed, as they are stand-alone CsgA and CsgB proteins in their Normal and Linker-modified versions (CsgA, CsgB, CsgAM, and CsgBM). The remaining samples in Table 1 (CsgAB, CsgAM-BM, CsgAM-B, and CsgA-BM) are the resultants of combinations involving the first four samples. One of the most important observations that can be made based on the measurements is that each sample that results from a combination of two proteins features a Young's modulus value that is larger than the individual Young's modulus values of its constituents. For instance, the $E_{\text {mean }}$ value for CsgAB $(11.6 \mathrm{MPa})$ is higher than the $E_{\text {mean }}$ value for both CsgA (9.5 $\mathrm{MPa})$ and $\mathrm{CsgB}(10.0 \mathrm{MPa})$. The same is true also for CsgAM$\mathrm{BM}$ (14.8 MPa vs 8.4 and 13.9 MPa), CsgAM-B (10.4 MPa vs 8.4 and $10.0 \mathrm{MPa}$ ), and, most significantly, for CsgA-BM (18.9 $\mathrm{MPa}$ vs 9.5 and $13.9 \mathrm{MPa}$ ), demonstrating that mechanically stiffer nanofibers can be deliberately obtained by combined secretion of major and minor proteins.

Additionally, it is interesting to note that the Normal group of samples shows a slightly increasing trend in the mean values of Young's modulus when progressing from CsgA, to CsgB, and to CsgAB. The increasing trend in the mean values of Young's modulus is seen to be preserved in the case of Linker-modified versions of the Normal samples (CsgAM, CsgBM, and CsgAM$\mathrm{BM})$. However, for all observations made in this section regarding mechanical properties, the statistical significance of the associated differences in Young's moduli need to be considered together with standard error of mean values, as discussed in section 3.3 and Figure 5.

As clearly demonstrated by the results of AFM force spectroscopy experiments presented so far, the mechanical properties of the amyloid nanofibers forming bacterial biofilms can be genetically tuned via the controlled secretion of proteins CsgA and $\mathrm{CsgB}$, as well as the optional Linker-modification process. A discussion of the related observations is presented in section 3.3.

3.3. Discussion of Tunable Mechanical Properties of Amyloid Nanofiber Structures. As demonstrated above, the mechanical properties of engineered biofilm structures can be tuned by controlling the expression of CsgA and CsgB proteins individually. This is also in good agreement with previous observations claiming that the secretion of CsgA and CsgB proteins individually changes the final morphology of biofilm structures according to the Congo red staining results. ${ }^{52}$ However, as seen in Figure 5a, we observed that there is not a significant difference between the mechanical properties of individual fibrils of $\operatorname{CsgA}$ and $\mathrm{CsgB}$ and their combination, CsgAB. The Young's modulus values of CsgA and CsgB nanofibers are similar which is not surprising considering their high sequence homology. These two proteins have similar assembly characteristics and similar structural features, as reported earlier. The molecular models of these proteins also support this conclusion (Figure 1b). As structural features are expected to dictate the mechanical properties of protein polymers, the experimentally observed similarity in the Young's modulus values of CsgA and CsgB is not surprising. Interestingly, the addition of $\mathrm{CsgB}$ to the $\mathrm{CsgA}$ protein and 


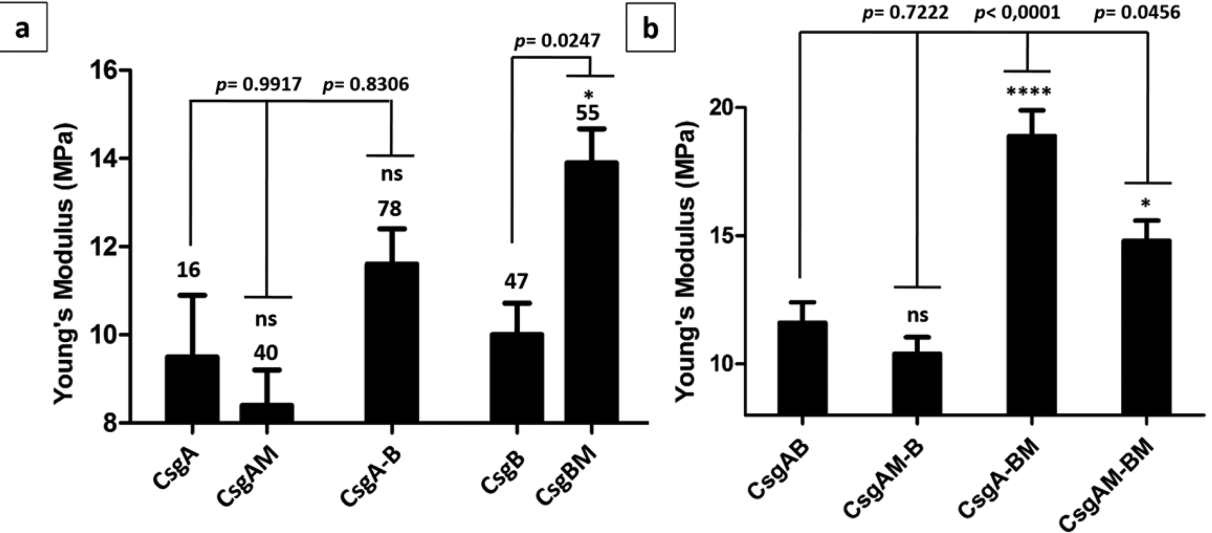

Figure 5. Comparison of Young's modulus values of major and minor proteins as well as their Linker-modified versions, along with statistical analysis. Error bars represent SEM (standard error of mean). The number of repeated measurements of Young's modulus is written above each column. (a) Two-way ANOVA and Tukey's post hoc comparisons reveal that Linker-modification significantly increases Young's modulus of the minor protein ( $\mathrm{CsgB}$ ) but not the major protein (CsgA). (b) One-way ANOVA and Tukey's post hoc comparisons reveal the significance of the Linker-modified minor protein in tuning the Young's moduli of amyloid fibers formed by both types of proteins. (ns, not significant; *, significant; $* * * *$, extremely significant; $p<0.05)$.

the formation of the native form of the biofilm backbone (CsgAB) does not cause a significant change in mechanical properties when compared to the mechanical properties of CsgA protein fibers, either (Figure 5a).

Following these observations, we genetically fused a Linker sequence to the CsgA protein (to obtain CsgAM) and a significant increase or change in its Young's modulus value was not noted (Figure 5a). On the other hand, fusing the same Linker sequence to the CsgB protein (to obtain CsgBM) triggered a statistically significant change in the Young's modulus, suggesting that the modified CsgB protein can play a key role in controlling the Young's modulus of the biofilm backbone formed by amyloid nanofibers (Figure 5a). To test this hypothesis, we compared the mechanical properties of the CsgAB nanofibers to the nanofibers obtained by replacing the native CsgA and CsgB with their Linker-modified versions. The results as given in Figure $5 \mathrm{~b}$, which shows that replacing the CsgA with its modified version CsgAM in the CsgAB backbone does not lead to a significant change in the mechanical properties of the final nanofiber structure, which is in agreement with our previous comparison: CsgA vs CsgAM. Replacing CsgB with its modified version CsgBM, on the other hand, results in a dramatic increase in the Young's modulus of the resulting CsgA-BM, which is statistically significant. Utilization of CsgAM along with CsgBM results in an increase of the Young's modulus value of the modified nanofiber structures (CsgAM-BM) when compared to the initial backbone (CsgAB), as well. Although this change is statistically significant, the Young's modulus is smaller compared to that of CsgA-BM. Moreover, it is observed that samples involving CsgBM result in higher Young's modulus values when compared to their counterparts involving CsgB. In particular, nanofiber structures formed by CsgA-BM are stiffer than those formed by CsgAB (18.9 $\mathrm{MPa}$ vs $11.6 \mathrm{MPa}$ ) and nanofiber structures formed by CsgAM-BM are stiffer than those formed by CsgAM-B (14.8 $\mathrm{MPa}$ vs $10.4 \mathrm{MPa})$. All of these results suggest that the Linker-modified CsgB protein (CsgBM) plays a key role in controlling the mechanical properties of the amyloid nanofiber structures.

It was previously reported that $\mathrm{CsgB}$ is crucial for the biofilm formation in E. coli as it plays a critical role during hierarchical self-assembly as a seeding molecule for CsgA. ${ }^{53,54}$ Our findings demonstrate that structural modification of the CsgB protein via Linker molecules can be used to significantly tune the mechanical properties of the resulting biofilm proteins. On the other hand, it is observed via Figure 3e that CsgBM by itself cannot form continuous biofilm backbone structures with high homogeneity. As such, there is a need to use it with the CsgA protein (in its normal or linker-modified states) to make robust biofilm backbone assemblies with continuous (as opposed to porous) morphologies (see Figure $3 f$ and Figure $4 d$ ).

It is very crucial at this point to clarify the potential physical reasons behind our observation of MPa-range Young's modulus values for the amyloid fibers in our study. While Young's modulus values in our study agree very well with several other works in the literature, ${ }^{43,44,55}$ it is to be noted that there are other studies that report Young's modulus values in the GParange for amyloid fibers, performed via various nanoindentation schemes. $^{45,56-59}$ Thereby, a subtle structural difference that would discriminate amyloid fibers exhibiting Young's moduli in the MPa-range from the ones in the GPa-range is to be noted. Specifically, the amyloids with Young's modulus values in the GPa-range seem to exhibit mostly defect-free, straight, tubular structures of defined diameters and packing geometries along their axial contours. ${ }^{45,56-59}$ That structural characteristic seems to be missing in the studies reporting MPa-range values for Young's moduli: The amyloid fibers with Young's modulus values in the MPa-range aggregate in more irregular and branched structures. ${ }^{43,44,55}$ The influence of these structural characteristics on measured mechanical stiffness is, for instance, evident in two separate works focusing on insulin amyloid fibers which report significantly different values for Young's modulus (5-50 MPa vs $\sim 3 \mathrm{GPa}$ ), whereby the fibers featuring more irregular structures also exhibit smaller Young's modulus values. $^{44,45}$ Therefore, when the highly branched and morphologically irregular structures formed by the bacterial amyloid nanofibers in our study are taken into account, the observation of MPa-range Young's modulus values is to be expected. Finally, as described in the literature, the additional constraints needed for packing longer and longer fibers may result in the destabilization of the whole amyloid structures, enabling preferential fractures to occur and thereby resulting in 
the growth of branched/aggregated fiber morphologies. ${ }^{55}$ It is to be expected that these structural defects would result in mechanical weakening, in exchange for increased branching and aggregation ability.

\section{CONCLUSIONS}

Bacterial biofilms are typically characterized as being involved in invasive microbial growth since they help microbial communities to sustain their states for longer time periods under harsh conditions. Bacterial functional amyloid nanofibers form the backbone of the biofilms in E. coli. The nanofibers are composed of two proteins: CsgA and CsgB. Functional amyloid nanofibers are large hierarchical self-assembled complexes. These protein complexes can be reprogrammed for biomedical and biotechnological applications. However, there is a lack of knowledge regarding the mechanical and biochemical aspects of the potential utilization of these proteins. Recently, there is a growing interest in controlling cellular function through mechanical signals. Developing new material systems with tunable mechanical properties will lead to the development of a new generation of biomaterials and scaffolds. Previously, nanofiber forming capacity of the bacterial biofilm proteins have been utilized in nanomaterial templating and growth. ${ }^{16,17}$ Given that protein-based nanoassemblies are promising candidates to be utilized as hybrid nanomaterials, ${ }^{60}$ bacterial biofilm protein can be used as new generation interfaces to synthesize long-range nanofibers of many different materials. In this study, we aimed to fill the gap with respect to our tuning capability regarding the mechanical stiffness of CsgA- and CsgB-enabled large protein assemblies, i.e., amyloid nanofibers. The results presented here demonstrate that (i) the Linkermodified CsgB protein can play a crucial role in controlling the mechanical properties of the resulting amyloid nanofiber structures and (ii) the CsgA protein (in its Normal or Linkermodified states) is needed for forming continuous, large-extent amyloid nanofiber structures. In essence, here we proposed a route to control the mechanical properties of bacterial functional amyloid nanofibers, which can be employed, for instance, as a scaffold for tissue engineering or as nanowires for future applications in materials science and nanotechnology.

\section{ASSOCIATED CONTENT}

\section{S Supporting Information}

The Supporting Information is available free of charge on the ACS Publications website at DOI: 10.1021/acs.langmuir.7b00112.

Details about AFM cantilever calibration and tip apex characterization, the experimental procedure involving FD spectroscopy, and genetic constructs and primers used in this study (PDF)

\section{AUTHOR INFORMATION}

\section{Corresponding Authors}

*(M.Z.B.) E-mail: mehmet.baykara@bilkent.edu.tr.

*(U.O.S.S.) E-mail: urartu@bilkent.edu.tr.

\section{ORCID}

Urartu Ozgur Safak Seker: 0000-0002-5272-1876

\section{Author Contributions}

The manuscript was written through contributions of all authors. All authors have given approval to the final version of the manuscript.

\section{Notes}

The authors declare no competing financial interest.

\section{ACKNOWLEDGMENTS}

U.O.S.S. thanks The Scientific and Technological Research Council of Turkey (TUBITAK) for financial support via Grant No. 114M163. M.T.A. and M.Z.B. thank TUBITAK for financial support via Grant No. 5140024. U.O.S.S. and M.Z.B. thank the Turkish Academy of Sciences for the Young Investigator Award (TUBA-GEBIP). E.K. acknowledges support by the TUBITAK-BIDEB Doctoral Fellowship Program. We thank Prof. Timothy K. Lu for the generous gift of E. coli $\Delta \operatorname{csgBA}$ strain. We thank Elif Ergül Duman for the preparation of statistical analysis of Young's modulus.

\section{REFERENCES}

(1) Jung, B. S.; Anvari, B. Virus-Mimicking Optical Nanomaterials: Near Infrared Absorption and Fluorescence Characteristics and Physical Stability in Biological Environments. ACS Appl. Mater. Interfaces 2013, 5 (15), 7492-7500.

(2) Jolley, C.; Klem, M.; Harrington, R.; Parise, J.; Douglas, T. Structure and photoelectrochemistry of a virus capsid-TiO2 nanocomposite. Nanoscale 2011, 3 (3), 1004-1007.

(3) Huggins, K. N. L.; Schoen, A. P.; Arunagirinathan, M. A.; Heilshorn, S. C. Multi-Site Functionalization of Protein Scaffolds for Bimetallic Nanoparticle Templating. Adv. Funct. Mater. 2014, 24 (48), 7737-7744.

(4) Fan, R. L.; Chew, S. W.; Cheong, V. V.; Orner, B. P. Fabrication of Gold Nanoparticles Inside Unmodified Horse Spleen Apoferritin. Small 2010, 6 (14), 1483-1487.

(5) Jorda, J.; Leibly, D. J.; Thompson, M. C.; Yeates, T. O. Structure of a novel $13 \mathrm{~nm}$ dodecahedral nanocage assembled from a redesigned bacterial microcompartment shell protein. Chem. Commun. 2016, 52 (28), 5041-5044.

(6) Xu, H.; Cao, B. R.; George, A.; Mao, C. B. Self-Assembly and Mineralization of Genetically Modifiable Biological Nanofibers Driven by beta-Structure Formation. Biomacromolecules 2011, 12 (6), 21932199.

(7) Wang, F. K.; Li, D.; Mao, C. B. Genetically Modifiable Flagella as Templates for Silica Fibers: From Hybrid Nanotubes to 1D Periodic Nanohole Arrays. Adv. Funct. Mater. 2008, 18 (24), 4007-4013.

(8) Adhikari, R. Y.; Malvankar, N. S.; Tuominen, M. T.; Lovley, D. R. Conductivity of individual Geobacter pili. RSC Adv. 2016, 6 (10), 8354-8357.

(9) Kumar, S. T.; Meinhardt, J.; Fuchs, A. K.; Aumuller, T.; Leppert, J.; Buchele, B.; Knupfer, U.; Ramachandran, R.; Yadav, J. K.; Prell, E.; Morgado, I.; Ohlenschlager, O.; Horn, U.; Simmet, T.; Gorlach, M.; Fandrich, M. Structure and Biomedical Applications of Amyloid Oligomer Nanoparticles. ACS Nano 2014, 8 (11), 11042-11052.

(10) Rambaran, R. N.; Serpell, L. C. Amyloid fibrils Abnormal protein assembly. Prion 2008, 2 (3), 112-117.

(11) Caspar, D. L. D. Inconvenient facts about pathological amyloid fibrils. Proc. Natl. Acad. Sci. U. S. A. 2009, 106 (49), 20555-20556.

(12) Romero, D.; Kolter, R. Functional amyloids in bacteria. Int. Microbiol. 2014, 17 (2), 65-73.

(13) Pham, C. L. L.; Kwan, A. H.; Sunde, M. Functional amyloid: Widespread in Nature, diverse in purpose. In Amyloids in Health and Disease; Perrett, S., Ed.; Essays in Biochemistry; Portland Press: London, 2014; Vol. 56, pp 207-219.

(14) Shewmaker, F.; McGlinchey, R. P.; Wickner, R. B. Structural Insights into Functional and Pathological Amyloid. J. Biol. Chem. 2011, 286 (19), 16533-16540.

(15) Chapman, M. R.; Robinson, L. S.; Pinkner, J. S.; Roth, R.; Heuser, J.; Hammar, M.; Normark, S.; Hultgren, S. J. Role of Escherichia coli curli operons in directing amyloid fiber formation. Science 2002, 295 (5556), 851-855. 
(16) Chen, A. Y.; Deng, Z. T.; Billings, A. N.; Seker, U. O. S.; Lu, M. Y.; Citorik, R. J.; Zakeri, B.; Lu, T. K. Synthesis and patterning of tunable multiscale materials with engineered cells. Nat. Mater. 2014, 13 (5), 515-523.

(17) Seker, U. O. S.; Chen, A. Y.; Citorik, R. J.; Lu, T. K. Synthetic Biogenesis of Bacterial Amyloid Nanomaterials with Tunable Inorganic-Organic Interfaces and Electrical Conductivity. ACS Synth. Biol. 2017, 6 (2), 266-275.

(18) Nguyen, P. Q.; Botyanszki, Z.; Tay, P. K.; Joshi, N. S. Programmable biofilm-based materials from engineered curli nanofibres. Nat. Commun. 2014, 5, 4945.

(19) Dai, H. X.; Choe, W. S.; Thai, C. K.; Sarikaya, M.; Traxler, B. A.; Baneyx, F.; Schwartz, D. T. Nonequilibrium synthesis and assembly of hybrid inorganic-protein nanostructures using an engineered DNA binding protein. J. Am. Chem. Soc. 2005, 127 (44), 15637-15643.

(20) Fan, R.; Chew, S. W.; Cheong, V. V.; Orner, B. P. Fabrication of gold nanoparticles inside unmodified horse spleen apoferritin. Small 2010, 6 (14), 1483-7.

(21) Zhong, C.; Gurry, T.; Cheng, A. A.; Downey, J.; Deng, Z.; Stultz, C. M.; Lu, T. K. Strong underwater adhesives made by selfassembling multi-protein nanofibres. Nat. Nanotechnol. 2014, 9 (10), 858-66.

(22) Botyanszki, Z.; Tay, P. K.; Nguyen, P. Q.; Nussbaumer, M. G.; Joshi, N. S. Engineered catalytic biofilms: Site-specific enzyme immobilization onto E. coli curli nanofibers. Biotechnol. Bioeng. 2015, 112 (10), 2016-24.

(23) Shu, Q.; Crick, S. L.; Pinkner, J. S.; Ford, B.; Hultgren, S. J.; Frieden, C. The E. coli CsgB nucleator of curli assembles to beta-sheet oligomers that alter the CsgA fibrillization mechanism. Proc. Natl. Acad. Sci. U. S. A. 2012, 109 (17), 6502-6507.

(24) Binnig, G.; Quate, C. F.; Gerber, C. Atomic force microscope. Phys. Rev. Lett. 1986, 56 (9), 930.

(25) Müller, D. J.; Anderson, K. Biomolecular imaging using atomic force microscopy. Trends Biotechnol. 2002, 20 (8), S45-S49.

(26) Müller, D. J.; Dufrene, Y. F. Atomic force microscopy as a multifunctional molecular toolbox in nanobiotechnology. Nat. Nanotechnol. 2008, 3 (5), 261-269.

(27) Santos, N. C.; Castanho, M. A. An overview of the biophysical applications of atomic force microscopy. Biophys. Chem. 2004, 107 (2), $133-149$.

(28) Müller, D. J.; Engel, A. Atomic force microscopy and spectroscopy of native membrane proteins. Nat. Protoc. 2007, 2 (9), 2191-2197.

(29) Dufrêne, Y. F. Towards nanomicrobiology using atomic force microscopy. Nat. Rev. Microbiol. 2008, 6 (9), 674-680.

(30) Drake, B.; Prater, C. B.; Weisenhorn, A. L.; Gould, S. A.; Albrecht, T. R.; Quate, C. F.; Cannell, D. S.; Hansma, H. G.; Hansma, P. K. Imaging crystals, polymers, and processes in water with the atomic force microscope. Science 1989, 243 (4898), 1586-1589.

(31) Butt, H.-J. Measuring electrostatic, van der Waals, and hydration forces in electrolyte solutions with an atomic force microscope. Biophys. J. 1991, 60 (6), 1438.

(32) Matzke, R.; Jacobson, K.; Radmacher, M. Direct, high-resolution measurement of furrow stiffening during division of adherent cells. Nat. Cell Biol. 2001, 3 (6), 607-610.

(33) Gaboriaud, F.; Dufrêne, Y. F. Atomic force microscopy of microbial cells: application to nanomechanical properties, surface forces and molecular recognition forces. Colloids Surf., B 2007, 54 (1), $10-19$.

(34) Müller, D. J.; Janovjak, H.; Lehto, T.; Kuerschner, L.; Anderson, $\mathrm{K}$. Observing structure, function and assembly of single proteins by AFM. Prog. Biophys. Mol. Biol. 2002, 79 (1), 1-43.

(35) Kalle, W.; Strappe, P. Atomic force microscopy on chromosomes, chromatin and DNA: a review. Micron 2012, 43 (12), 1224-1231.

(36) Müller, D. J.; Heymann, J. B.; Oesterhelt, F.; Möller, C.; Gaub, H.; Büldt, G.; Engel, A. Atomic force microscopy of native purple membrane. Biochim. Biophys. Acta, Bioenerg. 2000, 1460 (1), 27-38.
(37) Iyer, S.; Gaikwad, R.; Subba-Rao, V.; Woodworth, C.; Sokolov, I. Atomic force microscopy detects differences in the surface brush of normal and cancerous cells. Nat. Nanotechnol. 2009, 4 (6), 389-393.

(38) Gilbert, Y.; Deghorain, M.; Wang, L.; Xu, B.; Pollheimer, P. D.; Gruber, H. J.; Errington, J.; Hallet, B.; Haulot, X.; Verbelen, C.; Hols, P.; Dufréne, Y. F. Single-molecule force spectroscopy and imaging of the vancomycin/D-Ala-D-Ala interaction. Nano Lett. 2007, 7 (3), 796-801.

(39) Touhami, A.; Nysten, B.; Dufrêne, Y. F. Nanoscale mapping of the elasticity of microbial cells by atomic force microscopy. Langmuir 2003, 19 (11), 4539-4543.

(40) Hansma, P. K.; Schitter, G.; Fantner, G. E.; Prater, C. Highspeed atomic force microscopy. Science 2006, 314 (5799), 601.

(41) Fantner, G. E.; Barbero, R. J.; Gray, D. S.; Belcher, A. M. Kinetics of antimicrobial peptide activity measured on individual bacterial cells using high-speed atomic force microscopy. Nat. Nanotechnol. 2010, 5 (4), 280-285.

(42) Dokukin, M.; Sokolov, I. High-resolution high-speed dynamic mechanical spectroscopy of cells and other soft materials with the help of atomic force microscopy. Sci. Rep. 2015, 5, 12630.

(43) Knowles, T. P.; Buehler, M. J. Nanomechanics of functional and pathological amyloid materials. Nat. Nanotechnol. 2011, 6 (8), 469479

(44) Guo, S.; Akhremitchev, B. B. Packing density and structural heterogeneity of insulin amyloid fibrils measured by AFM nanoindentation. Biomacromolecules 2006, 7 (5), 1630-1636.

(45) Smith, J. F.; Knowles, T. P.; Dobson, C. M.; MacPhee, C. E.; Welland, M. E. Characterization of the nanoscale properties of individual amyloid fibrils. Proc. Natl. Acad. Sci. U. S. A. 2006, 103 (43), 15806-15811.

(46) Volle, C.; Ferguson, M.; Aidala, K.; Spain, E.; Nunez, M. Spring constants and adhesive properties of native bacterial biofilm cells measured by atomic force microscopy. Colloids Surf., B 2008, 67 (1), $32-40$.

(47) Beech, I. B.; Smith, J. R.; Steele, A. A.; Penegar, I.; Campbell, S. A. The use of atomic force microscopy for studying interactions of bacterial biofilms with surfaces. Colloids Surf., B 2002, 23 (2), 231247.

(48) Sader, J. E.; Larson, I.; Mulvaney, P.; White, L. R. Method for the calibration of atomic force microscope cantilevers. Rev. Sci. Instrum. 1995, 66 (7), 3789-3798.

(49) Kallberg, M.; Wang, H. P.; Wang, S.; Peng, J.; Wang, Z. Y.; Lu, $\mathrm{H}$.; $\mathrm{Xu}, \mathrm{J}$. B. Template-based protein structure modeling using the RaptorX web server. Nat. Protoc. 2012, 7 (8), 1511-1522.

(50) Tian, P. F.; Boomsma, W.; Wang, Y.; Otzen, D. E.; Jensen, M. H.; Lindorff-Larsen, K. Structure of a Functional Amyloid Protein Subunit Computed Using Sequence Variation. J. Am. Chem. Soc. 2015, 137 (1), 22-25.

(51) Reichhardt, C.; Jacobson, A. N.; Maher, M. C.; Uang, J.; McCrate, O. A.; Eckart, M.; Cegelski, L. Congo Red Interactions with Curli-Producing E. coli and Native Curli Amyloid Fibers. PLoS One 2015, 10 (10), e0140388.

(52) Hammer, N. D.; Schmidt, J. C.; Chapman, M. R. The curli nucleator protein, $\mathrm{CsgB}$, contains an amyloidogenic domain that directs CsgA polymerization. Proc. Natl. Acad. Sci. U. S. A. 2007, 104 (30), 12494-12499.

(53) Louros, N. N.; Bolas, G. M. P.; Tsiolaki, P. L.; Hamodrakas, S. J.; Iconomidou, V. A. Intrinsic aggregation propensity of the CsgB nucleator protein is crucial for curli fiber formation. J. Struct. Biol. 2016, 195 (2), 179-189.

(54) Hammer, N. D.; McGuffie, B. A.; Zhou, Y. Z.; Badtke, M. P.; Reinke, A. A.; Brannstrom, K.; Gestwicki, J. E.; Olofsson, A.; Almqvist, F.; Chapman, M. R. The C-Terminal Repeating Units of CsgB Direct Bacterial Functional Amyloid Nucleation. J. Mol. Biol. 2012, 422 (3), 376-389.

(55) Knowles, T. P.; Fitzpatrick, A. W.; Meehan, S.; Mott, H. R.; Vendruscolo, M.; Dobson, C. M.; Welland, M. E. Role of intermolecular forces in defining material properties of protein nanofibrils. Science 2007, 318 (5858), 1900-1903. 
(56) Adamcik, J.; Berquand, A.; Mezzenga, R. Single-step direct measurement of amyloid fibrils stiffness by peak force quantitative nanomechanical atomic force microscopy. Appl. Phys. Lett. 2011, 98 (19), 193701

(57) Adamcik, J.; Lara, C.; Usov, I.; Jeong, J. S.; Ruggeri, F. S.; Dietler, G.; Lashuel, H. A.; Hamley, I. W.; Mezzenga, R. Measurement of intrinsic properties of amyloid fibrils by the peak force QNM method. Nanoscale 2012, 4 (15), 4426-4429.

(58) Adamcik, J.; Jung, J.-M.; Flakowski, J.; De Los Rios, P.; Dietler, G.; Mezzenga, R. Understanding amyloid aggregation by statistical analysis of atomic force microscopy images. Nat. Nanotechnol. 2010, 5 (6), 423-428.

(59) Adamcik, J.; Mezzenga, R. Proteins fibrils from a polymer physics perspective. Macromolecules 2012, 45 (3), 1137-1150.

(60) Seker, U. O. S.; Mutlugun, E.; Hernandez-Martinez, P. L.; Sharma, V. K.; Lesnyak, V.; Gaponik, N.; Eychmuller, A.; Demir, H. V. Bio-nanohybrids of quantum dots and photoproteins facilitating strong nonradiative energy transfer. Nanoscale 2013, 5 (15), 7034-7040. 